\title{
Extensive exchange of transposable elements in the Drosophila pseudoobscura group
}

\author{
Tom Hill ${ }^{1 *}$ (D) and Andrea J. Betancourt ${ }^{2}$
}

\begin{abstract}
Background: As species diverge, so does their transposable element (TE) content. Within a genome, TE families may eventually become dormant due to host-silencing mechanisms, natural selection and the accumulation of inactive copies. The transmission of active copies from a TE families, both vertically and horizontally between species, can allow TEs to escape inactivation if it occurs often enough, as it may allow TEs to temporarily escape silencing in a new host. Thus, the contribution of horizontal exchange to TE persistence has been of increasing interest.

Results: Here, we annotated TEs in five species with sequenced genomes from the D. pseudoobscura species group, and curated a set of TE families found in these species. We found that, compared to host genes, many TE families showed lower neutral divergence between species, consistent with recent transmission of TEs between species. Despite these transfers, there are differences in the TE content between species in the group.

Conclusions: The TE content is highly dynamic in the D. pseudoobscura species group, frequently transferring between species, keeping TEs active. This result highlights how frequently transposable elements are transmitted between sympatric species and, despite these transfers, how rapidly species TE content can diverge.
\end{abstract}

\section{Background}

Transposable elements (TE) are parasitic mobile elements that propagate through the genomes of organisms, irrespective of the cost to the host [1-3]. TEs increase their numbers via transposition and the resulting non-Mendelian inheritance, but these factors are countered by suppression of transposition by hosts, by the generation of faulty, inactive copies during transposition, and by purifying selection acting against individual copies of a TE or against a TE family in aggregate [4-7]. Because of these inactivating forces, TEs may go extinct within a lineage, particularly if transposition rates become low due to host suppression [4, 8-10]. TE families can potentially temporarily escape this suppression by invading new hosts, with these horizontal transfers allowing them to persist in spite of extinction within individual lineages [11]. This process is exemplified by the recent horizontal transfer of the $P$-element, newly acquired by $D$. melanogaster sometime in the twentieth

\footnotetext{
*Correspondence: tom.hill@ku.edu

${ }^{1}$ The Department of Molecular Biosciences, University of Kansas, 4055

Haworth Hall, 1200 Sunnyside Avenue, Lawrence, KS 66045, USA

Full list of author information is available at the end of the article
}

century from a Caribbean species D. willistoni [12], followed by a further transmission into D. simulans [13, 14]. While copies of the P-element are typically highly degraded in $D$. willistoni, the element has been recently active in D. melanogaster and D. simulans [12]. Such horizontal transfer of TEs were once considered rare [12, 15], but have recently been shown to be pervasive, not just in Drosophila [5], but in other organisms as well [11, 16-18]. Transfer of TEs are thought to be more common among closely related species, and between those with overlapping geographic ranges $[18,19]$. Several transmission events have even been detected between hosts and parasites [19]. Other cases have resulted in changes that reshape the genome or generate phenotypic changes [11, 18], some of which resulted in adaptive changes, or changes involved in domestication [18, 20, 21].

The rates of horizontal transfer has implications for genome evolution. If horizontal transfer is rare, taxonomic groups may diverge in TE content over time, as individual TE families go extinct or are acquired by related species [4, 22-24]. If common, it may maintain active elements through regular exchange of active TEs between species

(c) The Author(s). 2018 Open Access This article is distributed under the terms of the Creative Commons Attribution 4.0 International License (http://creativecommons.org/licenses/by/4.0/), which permits unrestricted use, distribution, and reproduction in any medium, provided you give appropriate credit to the original author(s) and the source, provide a link to the Creative Commons license, and indicate if changes were made. The Creative Commons Public Domain Dedication waiver (http://creativecommons.org/publicdomain/zero/1.0/) applies to the data made available in this article, unless otherwise stated. 
[25]. These factors may go some way toward explaining differences between groups: for example, mammals, have few active TEs with mostly fixed insertions within species $[26,27]$, while in Drosophila TEs are highly active, as inferred from a high proportion of polymorphic insertions [28-32]. The forces of horizontal acquisition and suppression appear to lead to a slow, but detectable rate of turnover in TE content in the Drosophila genus: e.g. in the 12-genomes project [33], though all of the sequences species host Long Terminal Repeat (LTR), Long Interspersed Nuclear Elements (LINEs) retroelements and Terminal Inverted Repeat (TIR) DNA transposons [34], the proportion of the genome composed of repeats and the number of families appears to differ between species [33, 34].

Here, we examine the transposable element content in the D. pseudoobscura group in the Sophophora subgroup of Drosophila [33, 35-37]. This subgroup consists of four species with largely overlapping ranges, $D$. pseudoobscura, D. persimilis, D. miranda and D. lowei $[37,38]$. D. pseudoobscura was initially utilised as a study organism due to patterns of inversion polymorphism and variation in $\mathrm{Y}$ chromosome size [39-43]. These species are also able to hybridise to some degree in the lab [44-48], with D. pseudoobscura and persimilis showing little divergence outside of three fixed inversions between their genomes [45]. Unlike $D$. simulans and D. melanogaster, the D. pseudoobscura group species are not cosmopolitan [37, 40, 49] and thus may have had less opportunity to encounter new transposable elements due to range expansion or recent ecological changes. We use publicly available genome sequences for the four species, an outgroup species (D. affinis), and an improved genome sequence from $D$. pseudoobscura [33, $38,50]$. We use these data to examine changes in TE content among the species, and horizontal transfer of TEs within and from outside this group.

\section{Results and discussion}

TE annotation of the $D$. pseudoobscura group genomes

We identified TE families in the genomes of D. pseudoobscura, persimilis, D. miranda, D. lowei and D. affinis, and manually filtered and curated these sequences to generate 157 well-supported TE families found across the group (Fig. 1, Additional file 1: Figure S1). We also identified 15 sequences that pass all filters, but cannot be assigned to a $\mathrm{TE}$ order, these sequences were not included in further analyses (e.g. the 2 unknown sequences in D. pseudoobscura, Table 1, Additional file 2: Table S2), though they may represent undescribed TEs. Encouragingly, we found the 116 TE families previously described [51] for D. pseudoobscura using our pipeline, showing that our pipeline can independently recover the major families. We also found two TE families known from other Diptera species [51], and 28 additional putative TE families belonging to known orders that passed all our filters in these two species.

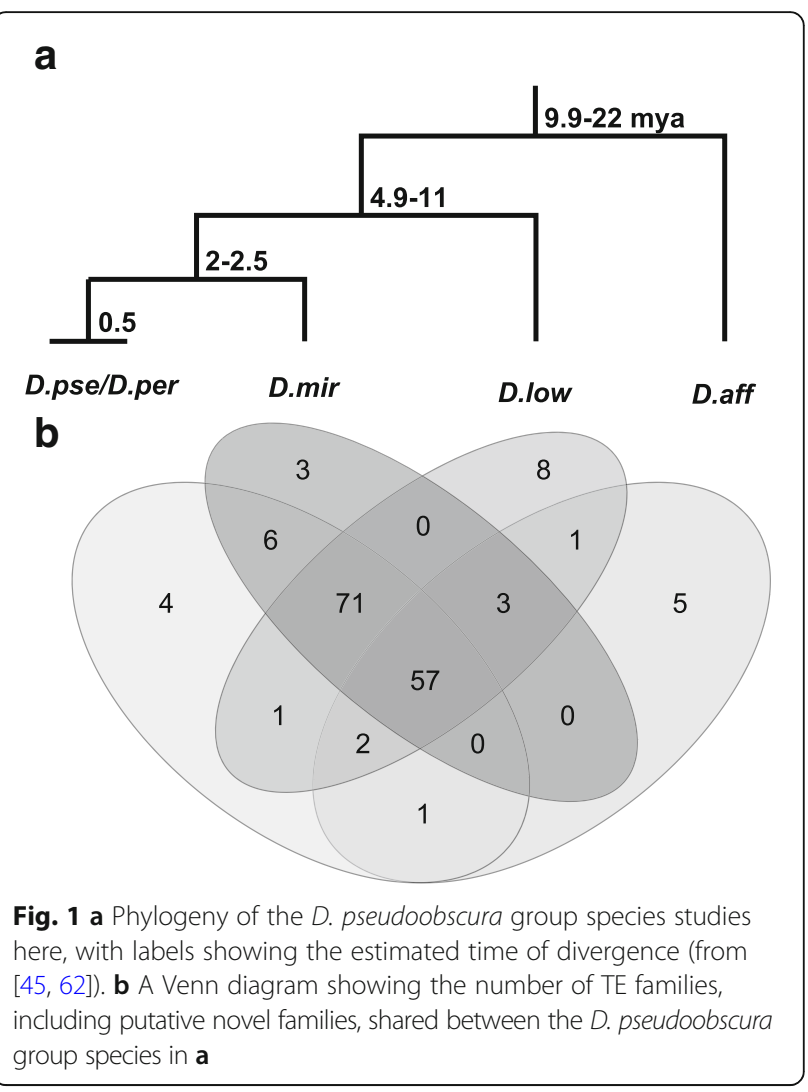

For D. pseudoobscura, we were able to use small RNA and RNAseq data $[52,53]$ to further support our annotations, particularly for the 28 putatively novel TEs. We used total body RNAseq data to estimate the fragments of mapped reads matching TE sequences (FPKM) for both novel and known TEs. Of the novel TEs, nine of 28 were expressed (Additional file 1: Figure S3, FPKM > 1), a similar proportion to that of the previously known TE families (49 of 116). Similarly, we found all 28 novel TE sequences and 114 known families had piRNAs generated against them (considering small RNA sequences 24-29 bp to be piRNAs). A subset of the piRNAs, those produced in the germline [7], are expected to show signatures of "ping-pong" amplification - small RNAs that match both sense and anti-sense strands of the TE sequence. These 'ping-pong' amplified sequences should also have a 10 bp overlap, a bias for uracil in the position 1 sense strand and adenosine bias at anti-sense position 10, due to their method of amplification $[9,54,55]$. We found that 60 elements (53 known families and 7 novel; 36 LTRs, 15 LINEs, 7 DNA transposons \& 2 helitrons) showed signatures of ping-pong amplification (Additional file 2: Table S2) [56]. Similar proportions of novel and known elements showed ping-pong small RNAs (Additional file 1: Figure S3, Mann-Whitney $U$ test $W=24, p$-value $>0.1676)$. As expression is difficult to quantify for multicopy sequences, these measures of expression are mainly useful to show 
Table 1 TE content of species in the D. pseudoobscura group

\begin{tabular}{|c|c|c|c|c|c|c|c|}
\hline \multirow[b]{2}{*}{ Species } & \multirow[b]{2}{*}{ TE Order } & \multirow[b]{2}{*}{ Families } & \multicolumn{3}{|l|}{ Reads } & \multirow{2}{*}{$\begin{array}{l}\text { Reference } \\
\text { Reference masked (\%) }\end{array}$} & \multirow{2}{*}{$\begin{array}{l}\text { PPTE2 } \\
\text { Num. ins }\end{array}$} \\
\hline & & & Percent reads & Est. copy number & dnapipeTE (\%) & & \\
\hline \multirow[t]{8}{*}{ D. pseudoobscura } & $\mathrm{TIR}$ & 31 & 1.745 & 414 & 1.65 & 0.98 & 292 \\
\hline & LTR & 72 & 8.875 & 2230 & 12.67 & 7.21 & 1846 \\
\hline & LINE & 35 & 3.633 & 1121 & 5.02 & 2.85 & 927 \\
\hline & $\mathrm{RC}$ & 3 & 1.852 & 978 & 2.83 & 1.21 & 978 \\
\hline & Polinton & 1 & 0.417 & 149 & 0.65 & 0.081 & 29 \\
\hline & Unknown & 2 & 0.332 & 22 & 0.8 & 0.017 & 6 \\
\hline & Total known & 142 & 16.522 & 4892 & 22.82 & 12.33 & 4072 \\
\hline & Total & 144 & 16.854 & 4914 & 23.62 & 12.5 & 4078 \\
\hline \multirow[t]{8}{*}{ D. persimilis } & $\mathrm{TIR}$ & 31 & 1.547 & 413 & 1.47 & 1.29 & 392 \\
\hline & LTR & 72 & 14.273 & 2260 & 15.24 & 12.95 & 1919 \\
\hline & LINE & 35 & 6.956 & 1301 & 6.92 & 5.76 & 958 \\
\hline & $\mathrm{RC}$ & 3 & 4.43 & 1781 & 4.11 & 3.41 & 1755 \\
\hline & Polinton & 1 & 0.034 & 46 & 0.49 & 0.18 & 46 \\
\hline & Unknown & 2 & 0.543 & 76 & 0.86 & 0.025 & 7 \\
\hline & Total known & 142 & 27.24 & 5801 & 28.23 & 23.59 & 5070 \\
\hline & Total & 144 & 27.78 & 5877 & 29.09 & 23.62 & 5077 \\
\hline \multirow[t]{8}{*}{ D. miranda } & $\mathrm{TIR}$ & 31 & 0.892 & 262 & 1.85 & 0.87 & 258 \\
\hline & LTR & 67 & 7.19 & 973 & 10.86 & 2.21 & 925 \\
\hline & LINE & 36 & 5.367 & 1431 & 9.26 & 1.25 & 1059 \\
\hline & $\mathrm{RC}$ & 5 & 1.484 & 1934 & 2.34 & 1.16 & 1934 \\
\hline & Polinton & 1 & 0.054 & 9 & 0.03 & 0.024 & 9 \\
\hline & Unknown & 2 & 0.337 & 4 & 0.27 & 0.015 & 4 \\
\hline & Total known & 140 & 14.987 & 4609 & 24.34 & 5.51 & 4185 \\
\hline & Total & 142 & 15.324 & 4613 & 24.61 & 5.53 & 4189 \\
\hline \multirow[t]{8}{*}{ D. lowei } & $\mathrm{TIR}$ & 31 & 1.396 & 495 & 1.46 & 0.382 & 381 \\
\hline & LTR & 74 & 6.883 & 1366 & 6.71 & 1.55 & 740 \\
\hline & LINE & 34 & 3.839 & 933 & 4.03 & 0.799 & 449 \\
\hline & $\mathrm{RC}$ & 5 & 1.245 & 813 & 1.83 & 0.363 & 523 \\
\hline & Polinton & 1 & 0.054 & 7 & 0.094 & 0.013 & 7 \\
\hline & Unknown & 9 & 0.641 & 265 & 3.9 & 0.087 & 241 \\
\hline & Total known & 145 & 13.417 & 3614 & 14.12 & 3.1 & 2100 \\
\hline & Total & 154 & 14.058 & 3879 & 18.024 & 3.187 & 2341 \\
\hline \multirow[t]{8}{*}{ D. affinis } & $\mathrm{TIR}$ & 9 & 0.872 & 278 & 3.25 & 0.177 & 230 \\
\hline & LTR & 47 & 4.328 & 630 & 8.4 & 1.427 & 832 \\
\hline & LINE & 13 & 5.223 & 530 & 6.4 & 0.406 & 339 \\
\hline & $\mathrm{RC}$ & 4 & 1.351 & 369 & 2.26 & 0.245 & 369 \\
\hline & Polinton & 1 & 0.068 & 35 & 0.77 & 0.041 & 35 \\
\hline & Unknown & 10 & 1.192 & 206 & 1.36 & 0.098 & 206 \\
\hline & Total known & 74 & 11.842 & 1842 & 21.08 & 2.29 & 1805 \\
\hline & Total & 84 & 13.034 & 2048 & 22.44 & 2.39 & 2011 \\
\hline
\end{tabular}

For each species, the table shows the number of TE families annotated for each order, and five metrics of TE content. These are: the proportion of Illumina reads mapping to TE sequences from each order (\% reads), the total number of copies from all families of that order, estimated from coverage relative to chromosome 3 (est. copy number), the proportion of the reference genome masked by each order, the proportion of reads (\% reads) per TE order as estimated by dnaPipeTE, and the number of insertions found using PopoolationTE2 [90]. As LTR elements often exist not as complete insertions, but as soloLTRs resulting from illegitimate recombination, coverage for the LTR elements was estimated for both solo LTRs and LTR bodies separately, with the mean taken across the combined sequences. We tested for extrachromosomal circular DNAs such as from Helitrons and Polintons via comparisons between copy numbers and insertion numbers 
that the putative novel TEs have characteristics similar to those of the known TE sequences (Mann-Whitney $\mathrm{U}$ test $\mathrm{W}=37, p$-value $>0.05$, Additional file 1: Figure S3), suggesting that they represent bona-fide TE sequences.

Unlike D. pseudoobscura and D. persimilis, there are no previous TE annotations for $D$. miranda, $D$. lowei and $D$. affinis. Most of the TE families we find in these species57 of 77-are shared among all five species of the D. pseudoobscura group (these sequences were independently verified in each species, and considered to represent the same family based on $\geq 90 \%$ sequence identity, Additional file 1: Figure S1). We also find 20 additional TE families in these newly annotated genomes (Fig. 1b, Additional file 2: Table S2). These new annotations are likely to be incomplete: TEs may be missed in genomes assembled exclusively from short read data, particularly if there is missing pericentric heterochromatin [34], or due to our filtering removing valid TEs. Consistent with this, the estimated proportion of TE content is higher for nearly all TE orders in all species when estimated using dnapipeTE [57], which does not rely on genome assembly (Table 1), than compared to our reference based annotations. We limit our analysis of TE content, therefore, to the reliable annotations of TE families from D. pseudoobscura and D. persimilis. For analysis of horizontal transfer, we use only well-described TE families; note that these analyses only require that the presence of a TE be detected in genome.

\section{TE content in the $D$. pseudoobscura group genomes}

We estimated overall TE content in the five sequenced species, and TE content broken down by order and family (Table 1, Additional file 2: Table S2). In particular, we quantified the TE content of our five focal species using five metrics: the proportion of the reference genome masked (using RepeatMasker [58]), the proportion of short reads mapping to each TE sequence, the proportion of short reads assembling to TEs using dnapipeTE [57], the number of insertions in each genome (called using PopoolationTE2 [59], demonstrated across genomes in Additional file 1: Figure S2) and the estimated copy number of each TE family (Table 1 and Additional file 2: Table S2). We find a significant linear correlation between all metrics at the level of TE order, and most metrics at the level of TE family (Spearman's rank correlation $p$-value $<0.00213$ ), with one exception: We find no linear correlation between the estimated copy number of TE families and the proportion of the genome masked by that TE family $(p>0.58)$. In contrast to a previous study, which found similar proportions of LTRs and LINEs in the D. pseudoobscura genome [33], we find LTRs are more than twice as abundant as LINEs (Table 1) as seen previously [34]. It is worth noting an additional effort was put into finding novel LTRs in the putative TE set using LTRHarvest [60], though downstream curation should have removed any false-positives.
Most of the TE content is due to the $57 \mathrm{TE}$ families shared across the entire group (73-84\% of insertions and $53-78 \%$ of each species reference TE content, Additional file 2: Table S2). Consistent with divergence in TE content between species over time, some TE families differ in copy number e.g. HelitronN-1 in D. miranda makes up $1.1 \%$ of the genome, while it only constitutes $0.14 \%$ of the $D$. lowei genome (Additional file 2: Table S2). These differences are possibly due to stochastic expansion degradation/extinction of families over time, or differing fitness costs between species. Specifically, in the case of HelitronN-1, we collapsed together Helitron $N-1$ and the closely related beneficial ISX sequence that has been co-opted for dosage compensation in $D$. miranda, as these have very similar sequences [61].

\section{Differences in TE content between species}

At first glance, $D$. persimilis is an outlier in the group, with much higher TE content than the other species in the $D$. pseudoobscura group (Table 1). However, while we annotated the $D$. miranda, $D$. lowei and $D$. affinis genomes using a pipeline identical to that for other species, there is good reason to believe we may have underestimated the TE content of these species, as discussed above (Table 1, Additional file 2: Table S2). In any case, the $D$. persimilis reference genome does appear to have approximately double the TE content of $D$. pseudoobscura, which is likely well-annotated here (23.59 versus $12.33 \%$ for the reference genome, Fig. 2a, Table 1). This level of difference is perhaps surprising for these closely related species thought to hybridise in nature $[45,46,62]$. A previous annotation from the 12-genomes project also found a similar $\sim 2$-fold enrichment in TEs for $D$. persimilis, although the estimated TE content was lower than that found here (3 and $8 \%$ vs. 12.33 and $23.59 \%$ here) [33].

As these species share the same TE families, any difference must be due to a difference in copy number; in fact, we found higher copy numbers in $D$. persimilis for most TE orders (Figs. 1 and 2, Table 1, Additional file 2: Table S2), implying a $21.3 \mathrm{Mbp}$ larger genome size in $D$. persimilis. While it is true that $D$. persimilis has a larger genome than D. pseudoobscura ([63], the genomes of females of the two species are estimated to differ only by $\sim 2 \mathrm{Mbp}$, [64, $65]$. The difference in copy number in the reference genomes may be partly due to the mixed male and female material used to construct the reference genomes. While considerable variation exists in D. pseudoobscura Y chromosome size between populations [41, 42], the $D$. pseudoobscura reference genome was likely generated from a strain containing the smallest $\mathrm{Y}$ chromosome type (Standard/Arrowhead, type V) [40, 41], and the D. persimilis from a strain with the most common $D$. persimilis $\mathrm{Y}$, which is cytologically the largest Y-chromosome type in the two species [41]. Thus, the $D$. persimilis reference likely 


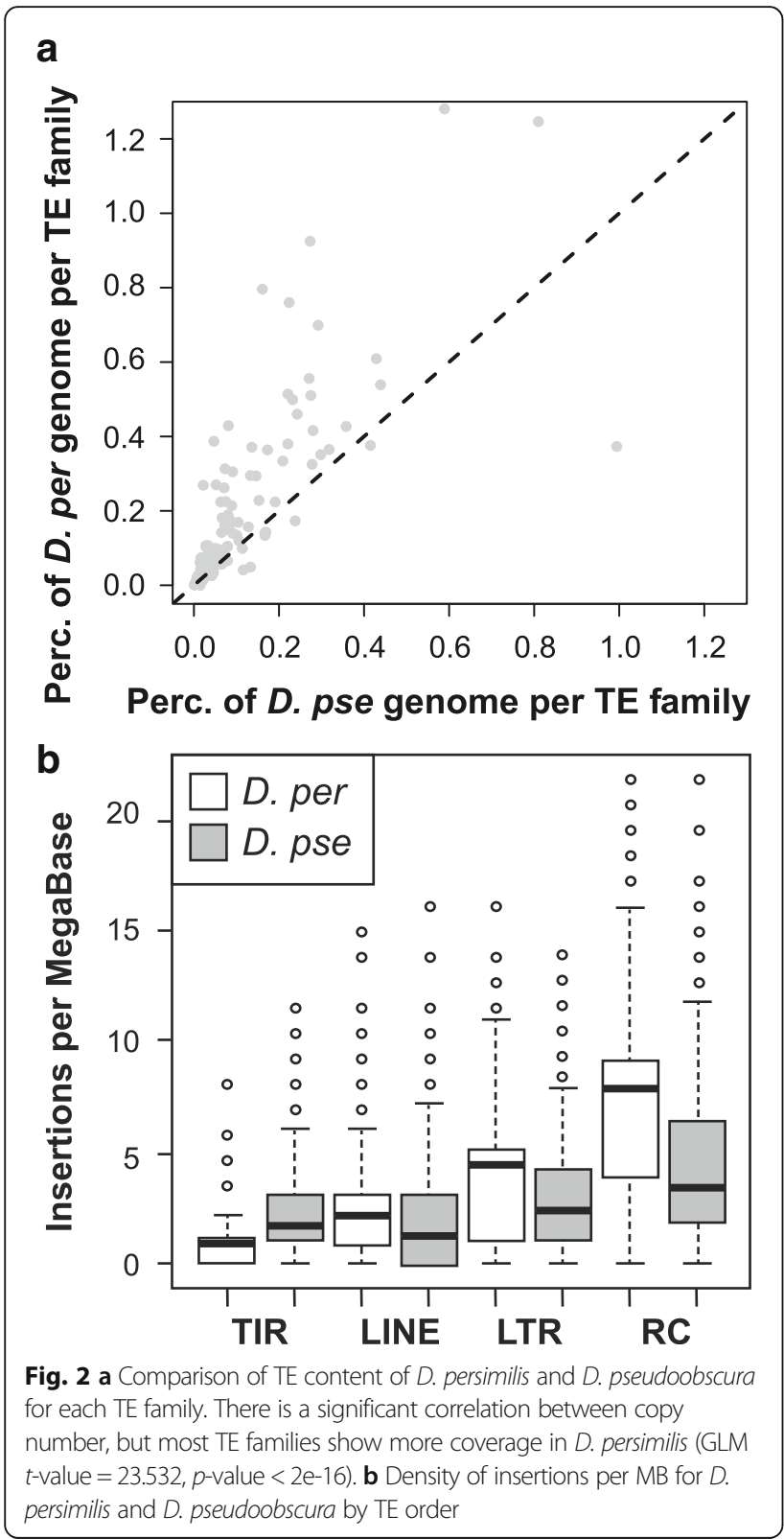

includes more TE-rich Y-chromosome sequence than the D. pseudoobscura one.

We therefore also estimated coverage from short read data which was collected exclusively from females and estimated the TE proportion with dnapipeTE. The bulk of the difference between $D$. persimilis and D. pseudoobscura seems due to a few families with large numbers of insertions in D. persimilis (e.g., Gypsy10_Dpse, HelitronN-1_Dpe, Gypsy17_Dpse, and MiniME_DP; Additional file 2: Table S2). Using these data, we estimate that $D$. persimilis has, at most, $\sim 5 \mathrm{Mbp}$ more TE content than $D$. pseudoobscura (from copy number and dnapipeTE [57] estimates), consistent with the minor differences in genome size found between the females of the two species [65].
Any difference in TE content between the species may in part be mediated by indirect effects of the very large Y-chromosome in $D$. persimilis, as in addition to being themselves TE-rich, Y-chromosomes may have indirect effects on TE content: Y-linked variation in D. melanogaster and $D$. simulans has been shown to be associated with TE regulation [66], with Y chromosomes apparently driving poor TE regulation due to the increased heterochromatin load in the genome [66-68].

We asked if, as for other differences between the species, these number differences are enriched in the paracentric inversions fixed between $D$. pseudoobscura and $D$. persimilis. Outside these regions, genetic differences are relatively homogenized between the species, likely due to, while inside these regions, divergence accumulates due to reduced genomic exchange [46]. We find a mixed effect of the inversions on TE copy number differences. For LTRs, copy numbers in inversions are elevated in $D$. persimilis, as expected when compared to outside inversions (Fig. 2b, Additional file 3, using inversion windows defined in [69]; Mann Whitney $U$ test: LTR insertions per $\mathrm{MB}$ inside inversions $\mathrm{W}=53,686, p$-value $=5.7 \mathrm{e}-05$, near inversions $\mathrm{W}=16,604, p$-value $=0.113$ and outside inversions $\mathrm{W}=290,520, p$-value $=0.141$ ). But inversions have little effect on copy numbers of $\mathrm{RC}$ and LINEs, which occur at higher density in D. persimilis genome-wide (Fig. 2b, Insertions per MB, Mann Whitney U test: $\mathrm{W}>335,780$, $p$-value $<0.0001$ for inside, outside and near inverted regions). These differences in the effect of inversion may reflect differences in timescales: LTR insertions tend to be young and highly polymorphic in Drosophila [70, 71], and thus should be affected by recent processes, such as post-speciation gene flow between these two species. We see no difference in TIR insertion densities (Fig. 2b, Insertions per $\mathrm{MB}$, Mann Whitney $\mathrm{U}$ test: $\mathrm{W}>$ $150, p$-value $=0.33$ ).

\section{Evidence of recent recurrent transfer between species within the $D$. pseudoobscura group}

Most TEs enter genomes vertically. Among those entering horizontally, a majority will enter from a closely related species in an overlapping range [11, 17-19, 67]. We looked at the rates of exchange of TE families between our focal species to assess the extent this contributes to the maintenance of active TE insertions. The geographic range of $D$. pseudoobscura overlaps with that of the other species studied here, apart from $D$. affinis and the subspecies $D$. pseudoobscura bogotona [72]. In addition, as mentioned previously, D. pseudoobscura and D. persimilis likely exchange genes through hybridisation, which is unlikely to occur in nature among the rest of the species in the group $[45,46]$. 
Following Bartholome et al. [25], we compared silent divergence between species at TE sequences to those for genes [69]; $d_{S}$ between species for recently horizontally transferred TE sequences will be low compared to that of vertically transmitted genes. To perform this analysis, we constructed a consensus sequence for each TE family for each species in the D. pseudoobscura group, and estimated synonymous site divergence $\left(d_{S}\right)$ using maximum likelihood [73] between these consensus sequences for each of the 10 species pairs.

We performed this analysis for $101 \mathrm{TE}$ families (those with previously described coding sequences), except for comparisons with $D$. affinis, where we used the 39 of TE families with annotated coding sequence of the 57 TEs found in all species. We found a significant overall reduction in $d_{S}$ for TEs compared to host genes for all species pairs (Fig. 3, Mann-Witney U test $p$-value $<0.05$ ), excluding those involving $D$. affinis (Mann-Witney $\mathrm{U}$ test $p=$ 0.23 , comparisons to D. pseudoobscura shown in Fig. 3a). We find 76 of the $101 \mathrm{TE}$ families (75.2\%) with $d_{S}$ that falls below that of the $2.5 \%$ quantile for nuclear genes in at least one comparison, suggesting potentially recent transmission between species ( 51 of 62 LTRs, 19 of 30 LINEs and 6 of 9 DNA transposons). Interestingly, $10 \mathrm{TE}$ families meet the criteria for horizontal transfer between all species pairs excluding those involving $D$. affinis $\left(d_{S}<0.25 \%\right.$ quantile: 1 TIR, 1 LINE and 8 LTRs), while 22 show no evidence of transfer $\left(d_{S}>50 \%\right.$ quantile: 1 TIR, 1 helitron, 11 LINEs and 9 LTRs), suggesting that families differ in their propensity for transfer. For species comparisons of similar levels of divergence (e.g. D. lowei-D. pseudoobscura, D. lowei-D. persimilis and D. lowei-D. miranda), we find no significant difference between rates of exchange (Man$\mathrm{n}$-Witney $\mathrm{U}$ test $p$-value $>0.08$ ). In addition to horizontal transfer, selection on silent sites can also depress $d_{S}$, providing an alternative explanation for the low $d_{S}$ of TEs [74]. We therefore re-examined the $d_{S}$ values using a method that controls for selection on silent sites due to selection on codon usage, VHICA (Vertical and Horizontal Inheritance Consistency Analysis) [74]. Consistent with our previous results, we find evidence of a high proportion of horizontally transferred families: 69 of 76 TE exchanges with low $d_{S}$ are identified as horizontal exchanges with $V H I C A$ as well ( $p$-value $<0.05$, Fig. $3 c$, with 1 LTR, 5 LINEs and 1 DNA transposon identified as vertically transferred instead). We also looked specifically for evidence of exchange between non-sister taxa, which would result in gene-tree/species-tree discordance: of the $76 \mathrm{TE}$ families with reduced divergence, phylogenies reconstructed for 42 are qualitatively inconsistent with the species tree (Figs. 1 and $3 \mathrm{~b}$ ). In principle, gene tree-species tree discordance could also be explained by incomplete lineage sorting, caused by TE sequences found in the common ancestor segregating into the descendant species such that TE with the most similar sequences are inherited by non-sister taxa. In this scenario, however, the TE consensus sequences should coalesce in the common ancestor, and thus would be expected to show high dS, not the low $\mathrm{d} S$ as seen here.

Transfer across species boundaries, in addition to happening by largely unknown mechanisms of horizontal transfer [20], can also occur via hybridisation. Within the D. pseudoobscura subgroup, D. pseudoobscura and $D$. persimilis, can produce fertile hybrids with others to some degree and are likely to regularly exchange genes in nature; (Machado et al. 2007), therefore, we cannot determine if these apparent transfer events are true horizontal events or hybridisation followed by introgression of TEs, but not genes. In fact, we do not find more evidence of transfer for LTRs and TIRs than LINEs, as is typical for genuine cases of horizontal transfer [20, 25], suggesting hybridisation as a mechanism of TE transfer in this group. That said, there is still ample evidence of exchange of TE families between species pairs that are sympatric [37, 47, 48, 75], but which cannot hybridise, e.g., D. pseudoobscura-D. lowei, D. pseudoobscura-D. miranda and $D$. miranda-D. lowei (Fig. 3b), and reduced $d_{S}$ between these species and D. pseudoobscura for TEs compared to genes (Fig. 3a, c).

In contrast, exchanges with the allopatric species in the group, $D$. affinis, there is little evidence of exchange, consistent with geographic isolation limiting opportunities for transfer (Fig. 3a, Mann Whitney $\mathrm{U}$ test: $p$ $<3.5 \mathrm{e}-08$, Additional file 2: Table S4). For TEs, $d_{S}$ between other focal species and $D$. affinis was significantly higher than for host genes (Fig. 3a, b). Additionally, we find no signatures of TE exchange using VHICA (Fig. $3 c)$. Thus, while we see abundant transfer between species that are sympatric, but not appear able to hybridise, the absence of exchange with $D$. affinis suggests routes of horizontal transfer that depend on proximity.

Under some scenarios, true horizontal transfer events allow TEs to escape host silencing, and are thus followed by bursts of transposition [9], which eventually subside as host silencing strengthens. We examined the TE sequence data for signatures of such bursts. For one, copy number expansion following horizontal transmission should be evident by an excess of low frequency single nucleotide differences between TE sequences, and thus a negative value of Tajima's D $[25,76]$. We estimated Tajima's D for each TE family in each species; in almost all cases, Tajima's D not significantly below zero (significance obtained from simulation [77]), suggesting no recent expansion in copy number (Fig. 3d). In contrast to results for the D. melanogaster group showing copy number expansion [25]. Interestingly, Tajima's D is slightly, non-significantly, lower in highly exchanging $\mathrm{TE}$ families compared to rarely exchanging $\mathrm{TE}$ families (Fig. 3d, t-test $p>0.57$ ). Similarly, bursts of 


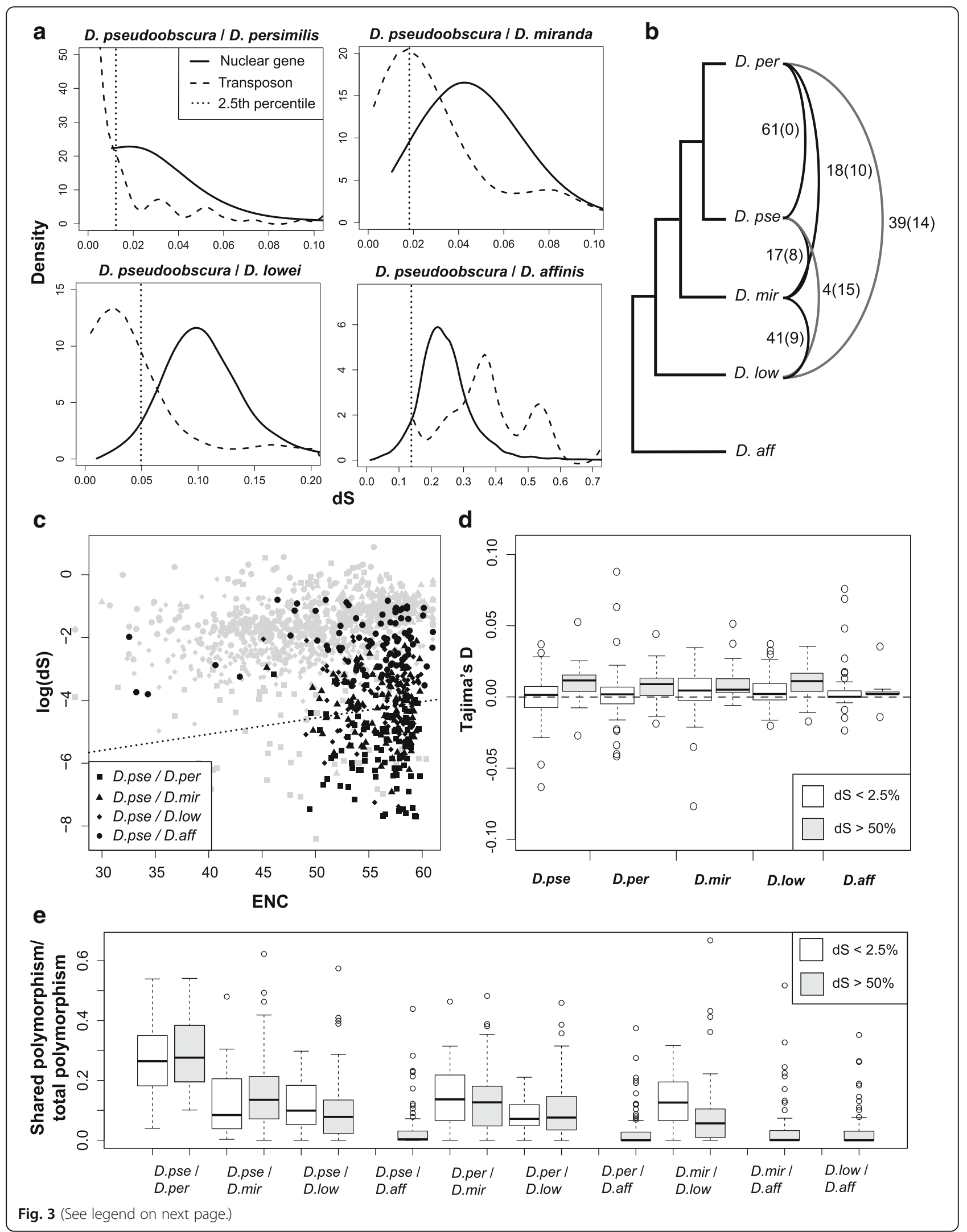




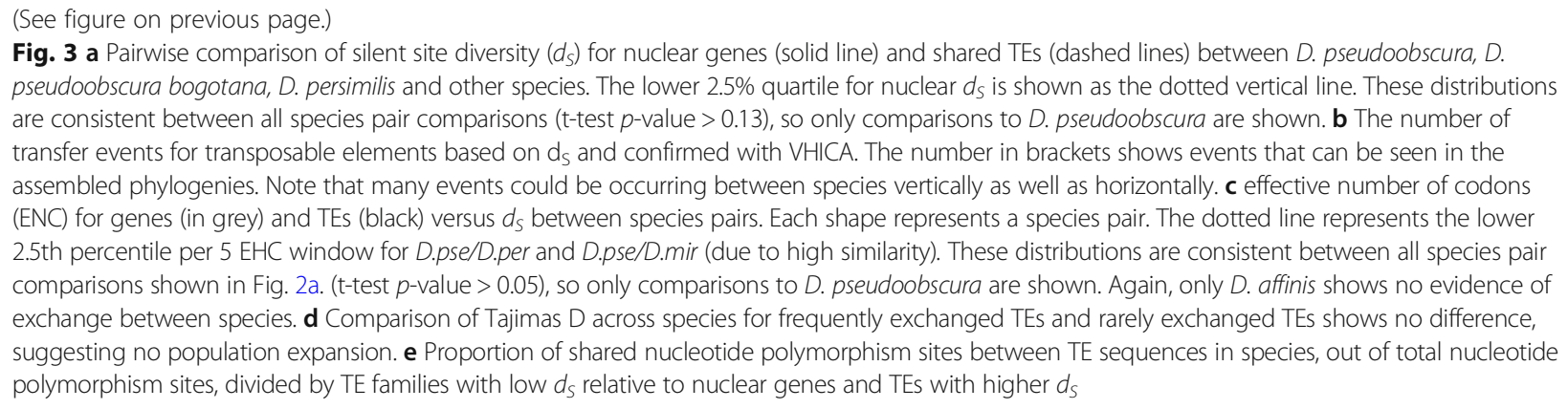

transposition would be expected to yield variation in copy number between species. In fact, we do see large variation in copy numbers for each family across species (Table 1, Additional file 2: Table S2). However, we compared the coefficient of variation, for pervasively transferring TEs, non-transferring TEs and all other TEs to ask if HT is associated with the differences in copy number between species. We find no difference in the coefficient of variation of copy number for pervasively transferring families and non-transferring families (Mann-Whitney $\mathrm{U}$ test, $p>0.19$ for all comparisons).

As an alternative to the horizontal transfer followed by burst model, these species may instead exchange TEs constantly (either via hybridisation or otherwise), so that any bursts following transfer are moderated. While previously we looked for a lack of divergence, further evidence for rampant horizontal exchange would be shared nucleotide polymorphisms across species boundaries, suggesting multiple TE sequences have been shared between species as opposed to a single shared copy (Fig. 3e, Additional file 2: Table S5). This suggests recurrent transmission between species, rather than single events $[25,76]$, or polymorphisms inherited from the TE in a common ancestor. Alternatively, there is less constraint on polymorphism in transposable elements, allowing recurrent mutation and polymorphisms to drift to higher frequencies in shorter periods of time following their horizontal acquisition. Generally, we find a negative correlation between synonymous divergence and shared polymorphism (significant for all comparisons apart from with $D$. affinis, $p<0.05$, no negative correlation between $D$. persimilis/D. pseudoobscura, Additional file 1: Figure S6).

These results together are likely due to a combination of gene flow between species in the wild, and recurrent horizontal transfer via other, as yet unknown, mechanisms, as in $[11,78,79]$. TE transfer following hybridisation may result in more homogenisation of TEs than of genes: while introgressed genes may be purged due to hybrid incompatibilities or genetic drift, their linked TEs may transpose readily after hybridisation, becoming unlinked from the introgressed genes. Particularly if accompanied by small RNA suppressors, these TE variants may be maintained in the new host with no accompanying change in Tajima's D [9]. Further, recurrent horizontal transfer between non-hybridising sympatric species of the pseudoobscura subgroup may recur frequently enough that TE families are freely shared between each of the species pairs, resulting in not only low $d_{S}$, but shared polymorphism and lack of copy number expansion (Additional file 1: Figure S6).

\section{Conclusion}

Like D. melanogaster, the D. pseudoobscura group shows highly active TEs that appear to be constantly undergoing a cycle of acquisition, expansion and high activity, suppression and finally extinction [4]. Surprisingly, despite TE exchange between species, the group shows distinct differences in TE content and TE densities consistent with high activity and turnover. Some of these differences may due to differences in quality of assembly of each species genome and method used to identify TE insertions. We find a distinct expansion in TE numbers in D. persimilis potentially due to differential regulation of TEs. Overall this suggests that despite frequent gene flow, TE dynamics can evolve rapidly across the lifetime of a TE family.

Due to the history of the first recorded instance of a horizontal transfer of a transposable element [12, 80, 81], we previously thought these transfers are rare and likely catastrophic events. However, an expanding body of evidence suggests that these events are likely a common occurrence throughout genomes, becoming more and more common the more closely related two species are $[17,25]$. This transfer of elements is possibly even recurrent in some cases. Our results support the view that the TE content of genomes is fluid, with TEs moving between genomes easily, with only occasional catastrophic events such as the invasion of the P-element.

\section{Methods}

\section{Sequence data}

We used publicly available reference genomes for five species: D. pseudoobscura (NCBI: PRJNA18793), D. persimilis (NCBI: PRJNA29989 genome assembled from Sanger sequence reads, http://popoolation.at/persimilis_genome/ for the genome based on illumina reads), D. affinis reads from 
(NCBI: ERX103526) and assembly (http://popoolation.at/ affinis_genome/), D. lowei (http://popoolation.at/lowei_genome/; Palmieri et al. 2014), D. miranda (NCBI: PRJNA77213) All sequence data used is summarized in Additional file 2: Table S1. We also used publicly available paired-end illumina data from inbred lines for four of these species [D. persimilis (SRA: SRR330426), D. miranda (SRA: SRR1925723), D. lowei (SRA: SRR330416 and SRR330418) and D. affinis (ENA: ERR127385)]. As we were unable to find publicly available paired-end illumina data for D. pseudoobscura, we used a data generated from an individual wild $D$. pseudoobscura made homozygous for the reference third chromosome inversion type (SRA: SRR617430; Fuller et al. 2016). As a result, only the third chromosome represents a wild chromosome, the rest of the genome is a mosaic of material from the wild and from the two different balancer stocks used.

\section{De novo annotation of transposable elements in the $D$. pseudoobscura group}

We annotated TE families in all five species, as well as putative TE sequences in the more diverged species (such as D. lowei and D. affinis), and compared our de novo annotations to the previous annotations for $D$. pseudoobscura and $D$. persimilis. These sequences were identified using RepeatModeler and LTRHarvest [60, 82]. Due to potential false positives called by these tools, we further filtered sequences, as outlined in Additional file 1: Figure S1 to give us a set of 'high confidence' TE annotations confirmed across several species.

To de novo annotate the transposable elements, as shown in Additional file 1: Figure S1:

1. We recovered a set of TE candidates for each species using the reference genomes. We used two separate pipelines: (i) Repeatscout and PILER in the RepeatModeler pipeline (default parameters) [82, 83], with all sequences designated as microsatellites and simple repeats removed from the output, and (ii) LTRHarvest, which finds LTR retrotransposons (using parameters recommended in the LTRHarvest manual: -tis -suf -lcp -des -sds - dna; -seed 100 -minlenltr 100 -maxlenltr 1000 -mindistltr 1000 -maxdistltr 15,000 -xdrop 5 -mat 2 -mis -2 -ins - 3 -del -3 -similar 90.0 -overlaps best -mintsd 5 -maxtsd 20 -motif tgca -motifmis 0 -vic 60 -longoutput) [60]. Though this step may bias us to find primarily LTRs, we note that most previously known TEs we find are LTRs, while most (19 of 41) novel elements are DNA transposons (Additional file 2: Table S2).

2. Step 1 resulted in a set of 769 candidate TE sequences, ranging from $208 \mathrm{bp}$ to $14.5 \mathrm{~kb}$. We used BLAST to filter and annotate the candidate TEs (parameters: e-value $<1 \mathrm{e}-08,-$ word_size 10 , -perc_identity 85) [84], by searching a database of all known Repbase and Flybase transposable element sequences for Diptera (including 121 TEs previously found in D. pseudoobscura, $D$. persimilis or D. miranda), with sequenced duplicated between the data bases removed using a custom python script.

a. Sequences that show single BLAST hits (e-value $\leq 1 \mathrm{e}-08)$ to this data base were assumed to represent a previously identified TE family. We discarded these sequences and used the Repbase/Flybase TE sequence to represent the family instead. (349 sequences).

b. From the remaining sequences, those that showed BLAST hits to several TE families, all from one superfamily, were considered to potentially represent a previously unidentified family within that superfamily. (180 sequences).

c. Of the remaining sequences, those with hits all in a single order, but to multiple superfamilies, were potentially novel TEs within this order. (18 sequences).

d. For sequences which had no potential TE family assigned in Step 2 (222 sequences), we attempted to find matches by aligning them to the online NCBI non-redundant database using megablast. Of these, 202 had annotated or predicted genes as the primary BLAST hit; these were discarded. The remaining potentially novel TEs were retained (20 sequences),

3. To facilitate downstream analysis, we obtained a single representative sequence for the potential novel TEs identified in Steps 2b, c and d, as is already done for those in Step 2a. To do this, we clustered sequences found for all species using vmatch (recommended LTRHarvest parameters: -dbcluster 957 -p -d -seedlength 50 -1 1101 -exdrop 9) [85]. We confirmed these clusters by BLASTing novel TE sequences to themselves and grouping them by similar matches (parameters: e-value $<0.00001$, -word_size 10 ).

4. As these may only represent partial TE sequences, we further assembled the grouped sequences using Trinity (default parameters) to collapse similar sequences and get a representative sequence for the cluster, even if only a fragment of the consensus sequence [86]. We checked these assemblies and clusters by aligning sequences from the cluster and with the Trinity assembly (if applicable) using MAFFT (parameters: -thread 3 -threadit 0 -reorder -leavegappyregion -auto) [87], to ensure that the assembly or longest sequence representing the putative novel TE was recovered. From each cluster of similar sequences, we took the longest 
sequence as the representative fragment of each putatively novel family.

5. Some of the putatively novel families identified in $2 \mathrm{~b}$ may instead be divergent representatives of known families. To see whether this was the case, we again attempted to identify previously known families among them using the consensus sequences from the five species genomes. We aligned novel TEs pairwise to all Repbase TEs using MAFFT (parameters: -thread 3 -threadit 0 -reorder -leavegappyregion -auto) and used a custom python script to find the number of diverged aligned bases. We defined sequences as belonging to a known family if they were $>90 \%$ similar to a known family across the sequence, following [51]. Two families of the novel sequences were found to belong to known families in this way (an Ielement and a Jockey element), but were closely related to insertions in distant relatives of the obscura group (I-4_DF from D. funebris and Jockey-8_DRh from D. rhopaloa, respectively). We therefore retained these sequences in our data set, as they likely represent diverged copies of these families, or ancient horizontal acquisitions.

6. From Steps 1-5, we found 567 candidate TE sequences, 349 of which belong to previously described TE families, including all 121 families previously found in the D. pseudoobscura group ('known' families), and 446 others (putative 'novel' families). We proceeded to filter sequences from this set which were represented by very few or very short matches to the reference genomes.

a. First, we used the 567 sequences to repeat mask the reference genome of each species using RepeatMasker (parameters: -no_is -norna no_low -gff -gccalc -u -s -cutoff 200) [58], following recommendations in [70]. We required that the families have at least 25 Repeatmasker hits in at least one species (237 sequences retained, 116 known and 121 novel families).

b. We then estimated the copy number of each TE family for each species from the Illumina short read data from adult females, discarding those estimated to have a median coverage less than 2-fold that of the third chromosome for less than $80 \%$ of the length of the sequence. To do this, we mapped short reads to the repeated masked reference genome and the 237 TE sequences retained from the previous step using BWA MEM (parameters: paired end -t $5-\mathrm{M}$ ) [88], and estimated coverage with bedtools genomecov [89]. Due to the poor assembly of the $D$. persimilis genome, we used a reference consisting of the $D$. pseudoobscura genome and the D. persimilis TE sequences. (157 sequences retained, from 116 known and 41 families novel to this species group).

We considered these 157 sequences to be an adequate representation of the TE content in the pseudoobscura group, though we recognize that our conservative approach may have discarded some true TE sequences.

Using this method, we found strong support for 114 of the $121 \mathrm{TE}$ families previously described in D. pseudoobscura, D. persimilis or D. miranda and 2 TEs previously identified in other Diptera species. We found 41 putatively novel sequences, including two subfamilies of previously known sequences, 30 newly assembled sequences which BLAST exclusively to one super family, and nine potentially new families that BLAST to one TE order. We also found 15 sequences that cannot be assigned an order (either due to BLAST hits to multiple orders, or no BLAST hits). These 15 sequences passed all filters, including being found multiple times in species genomes and did not correspond to genes or other NCBI sequences in a non-redundant BLAST search. To avoid unreliable inferences, we discarded these sequences from downstream analyses, but gave each of the 41 novel sequences an ID (Additional file 2: Table S2), and included them in masking and mapping stages. Sequences are available in Additional file 4.

For an independent verification of TE content, unbiased by reference genome, we generated dnapipeTE [57] profiles for each species using illumina sequencing information (-genome_coverage 0.5 -sample_number 2 -genome_size previously estimated size). We compared the proportions of each TE order in the genome to our referencegenome estimates and the proportion of reads mapping to TE sequences.

\section{Estimating TE density in the reference genome}

We used RepeatMasker v. 4.0.6 to mask each reference genome using the 157 consensus TE sequences and 15 unknown sequences from the de novo annotation, (parameters: -no_is - norna - nolow - gff - gccalc $-\mathrm{u}-\mathrm{s}-$ cutoff 200) [58]. To estimate the TE density of each genome, we calculated the density of TE bases per $1 \mathrm{MB}$ sliding window (with a step size of $100 \mathrm{~kb}$, after removing all $\mathrm{N}$ bases [e.g. TE bases / [window size - Ns in chromosome]]), across both assembled scaffolds and unassembled contigs from each reference genome.

\section{Identifying insertions in reference genomes and in sequenced third chromosome lines of $D$. pseudoobscura} To identify insertion sites in the reference genomes of D. pseudoobscura and D. persimilis, we used the 
PopoolationTE2 pipeline [90]. We chose to use the D. pseudoobscura masked reference, rather than the fragmented $D$. persimilis reference, as it facilitated mapping reads to genomic insertion sites. We expect similar results as these species are closely related $(0.018$ average synonymous divergence [45]), and we find that a similar proportion of reads map to TEs regardless of whether the $D$. pseudoobscura or D. persimilis genome is used (27.63 vs $27.27 \%$ ).

We then mapped available Illumina reads to the repeat masked references, the consensus TE sequences, and to sequences matching these consensus TEs identified by RepeatMasker using BWA-MEM (parameters: paired end $-t 5-M$, with secondary alignments reported, but marked) [88]. Using masked TE sequences to aids mapping of degenerate TE sequences, as described in [90].

Following mapping, we generated a ppileup file summarizing identities and locations of TE insertions for all lines in PopoolationTE2 (default settings, --map-qual 10 ) and subsampled to a physical coverage of 25 , removing secondary alignments. As these sequences are mostly from inbred lines, we required the estimated frequency to be at least $50 \%$ (default parameters, --target-coverage 25 , - -min-count 5 , minimum frequency $=0.5$ ) [90]. We then identified the number of insertions per MB window (after adjusting for the number of $\mathrm{N}$ bases in the window [e.g. TE number / [window size $-\mathrm{Ns}$ in window]]) across the genome of each species.

\section{Expression confirmation of putative TE sequences}

To see which TEs showed evidence of expression, we used RNAseq data for mRNAs (SRA: SRR1956914, taken from [52]) and small RNAs (SRA: SRR032435, taken from [91]) from the D. pseudoobscura reference line (MV-25). Before further analysis, we trimmed all genomic and RNAseq Illumina reads used with Sickle to remove low quality sequence data (default parameters for long reads, minimum length $=16$ for small RNAs, 50 for mRNAs), and removed reads that were unpaired (apart from the small RNA reads) after this step from the sequence data [92].

We mapped small RNA sequences from D. pseudoobscura to known and novel TEs identified in that species, using publicly available small RNA reads from the reference strain ([91], SRA: SRR032435). We first removed non-TE related small RNAs, following [7, 93], by mapping to a database of known Drosophila viruses and small RNAs other than those that are TE-related, including miRNAs, viral siRNAs, snoRNA [93], using BWA aln and allowing for up to 3 mismatches (parameters: $-n$ 3) [7, 88]. We then mapped the remaining reads to the repeat masked $D$. pseudoobscura reference genome and the novel and known TE sequences identified in this study ( $B W A$ aln parameters: -n 3 , maximum 2 alignments). We classified small RNAs by length and orientation using a custom python script and the Pysam python library, following [94]. Specifically, we considered small RNAs from 21 to 23 to be siRNAs and from 24 to 29 to be piRNAs [95]. We used bedtools (intersect, -wa -wb -f $0.3-\mathrm{r}$ ), to check for a 10-bp overlap between sense and anti-sense matches and used sequence logos [56] to check for the 1-T, 10-A bias, both associated with ping-pong amplification, a characteristic feature of piRNAs [96].

\section{Detecting short range horizontal transfer events within the pseudoobscura group}

To detect horizontal transfer of TEs within the five species examined, we compared divergence between consensus TE sequences to genomic divergence, following the rationale described in [25]. We limited this analysis to families found in at least 3 species and with an annotation on Repbase. As this method requires consensus sequences, we constructed these for each TE family and each species, we identified the major allele for each species at each variable site using GATK v3.5-0-g36282e4 HaplotypeCaller, with ploidy levels set to the estimated copy numbers based on coverage of the TE sequence, and using FastaAlternateReferenceMaker (default parameters) to generate fasta sequences from the mapped data [97]. We aligned these consensus sequences from each species using MAFFT (parameters: -thread 3 -threadit 0 -reorder -leavegappyregion -auto) [87] and generated a phylogeny of each sequence using the Repbase annotation and PhyML (parameters: -M GTR) [98]. We obtained a total of 39 annotated alignments that included sequences for $D$. affinis comparisons, and 62 additional sequences for all other pseudoobscura group species comparisons (noted in Additional file 2: Table S2).

We estimated synonymous site divergence $\left(d_{S}\right)$ in the TE sequences pairwise between species using codeml (with transition-transversion rates estimated from the data, and codon frequencies from the nucleotide frequencies) and the coding regions for these TEs as annotated in Repbase [51, 73]. We then compared $d_{S}$ of TEs to that of orthologous genes between species obtained in the same way, taken from Avila et al. (2014). Following Bartolomé et al. (2009), we considered an individual family to show strong evidence of exchange if its $d_{S}$ value was below the $2.5 \%$ quantile of the $d_{S}$ of all nuclear genes, to have potentially transferred if $d_{S}$ was between the 2.5 and $50 \%$ quantiles, and to show no evidence of transferring if above the $50 \%$ quantile.

We used the VHICA pipeline to confirm these putatively horizontal transfers, estimating the effective number of codons for each TE coding sequence and gene sequence for each species using the VHICA R package [74]. We considered a TE to have horizontally transferred with a significant $p$-value from the VHICA $\mathrm{R}$ package and a $d_{S}$ below two standard deviations of the genic mean, per ENC [74]. 
We examined polymorphism within TE families for evidence of horizontal transfer. We estimated Tajima's $D$ of each TE using Popoolation [99], with the TE copy number as the sample size. As negative Tajima's D may reflect recent expansion of a TE family [25]. We compared the levels of polymorphism shared among TEs in each species between potentially transferred TEs $\left(d_{S}<\right.$ $2.5 \%$ quantile) and TEs that are unlikely to have transferred $\left(d_{S}>50 \%\right.$ quantile). Using known estimates of Watterson's theta for D. pseudoobscura, we calculated the expected neutral distribution of Tajima's D for 1000 simulations in $m s$ [77].

\section{Additional files}

Additional file 1: Figure S1. Pipeline for TE annotation. Figure S2. TE density across the genomes of each species, found using PopoolationTE2, sorted by TE order. Figure S3. Comparison between putatively novel and known TE sequences for (A) length, (B) expression, (C) small RNA silencing expression and (D-F) copy number. Figure S4. Distribution of TE copy numbers per species. Figure S5. Phylogenies of each TE super family including novel TE families, used to calculate patristic distances. Figure S6. Correlation between silent substitutions in TEs between species and the proportion of silent shared polymorphism between species. (DOCX $1531 \mathrm{~kb}$ )

Additional file 2: Table S1. D. pseudoobscura species group lines used in this study. Table S2. TEs found in D. obscura group. Sorted by if they are previously discovered or novel, then by Order and super family. Transmission states if the TE family is found to transfer between species. Table S3. Diagonal table showing the total number of TE families found in each species for comparison. In brackets, the number of novel TE families found shared between species. Table S4. Comparisons of dN/dS between TEs and nuclear genes. The dS values presented here are compared to the dS values of nuclear genes between the given species calculated previously. We considered a transfer event between two species to have occurred if the TE dS value is less than the 2.5th percentile for nuclear genes. For instances where no dS for nuclear comparisons are available, we used the dS between D. pseudoobscura and the species of interest. Table S5. Number of unique and shared polymorphic sites for each species comparison, for each TE family, used in the boxplots in Fig. 2e. (XLSX $153 \mathrm{~kb}$ )

Additional file 3: TE insertion density per megabase (estimated from PopoolationTE2 output) for each TE order and each species analysed here. (TXT $166 \mathrm{~kb}$ )

Additional file 4: Fasta file of TE sequences generated in the TE annotation, with basic description of each TE sequence. (TXT $892 \mathrm{~kb}$ )

\section{Abbreviations}

FPKM: Fragments per kilobase of gene per million reads; LINE: Long interspersed nuclear element; LTR: Long terminal repeat; RC: Rolling circle; TE: Transposable element; TIR: Terminal inverted repeat

\section{Acknowledgements}

The authors acknowledge L. Endler and D. Gómez Sánchez for discussion and advice concerning the bioinformatic analysis. We are grateful for helpful discussion provided by K. Senti, R. Kofler, B. Charlesworth, A. Clark, R. Unckless and J. Blumenstiel. Thanks for S. W. Schaeffer and M. Noor for providing the data used in this survey and advice concerning how the data should be used. The authors thank the suggestions of three anonymous reviewers who helped shape and hone the manuscript.

\section{Funding}

This work was supported by a postdoctoral fellowship from the Max Kade foundation (Austria) to $\mathrm{TH}$, and by grant P27048 from the Austrian Science Fund (FWF) to AJB.

\section{Availability of data and materials}

All data used in this study (summarized in Additional file 2: Table S1) is freely available online through the NCBI short read archive (NCBI SRA: ERR127385, SRR330416, SRR330418, SRR1925723, SRR330426, SRR330420, SRR330423, SRR617430-74, SRR032435, SRR1956914). All genomes used are either available through flybase.org or popoolation.at.

\section{Authors' contributions}

TH performed bioinformatics analysis, statistical analysis. AB developed methods and analyses used. Both authors contributed to, read and approved the manuscript.

Ethics approval and consent to participate

Not applicable.

Competing interests

The authors declare that they have no competing interests.

\section{Publisher's Note}

Springer Nature remains neutral with regard to jurisdictional claims in published maps and institutional affiliations.

\section{Author details}

The Department of Molecular Biosciences, University of Kansas, 4055 Haworth Hall, 1200 Sunnyside Avenue, Lawrence, KS 66045, USA. ${ }^{2}$ Institute of Integrative Biology, University of Liverpool, Liverpool L69 7ZB, UK.

Received: 22 March 2018 Accepted: 1 June 2018

Published online: 19 June 2018

References

1. McClintock B. Induction of instability at selected loci in maize. Genetics. 1953;38:579-99.

2. Orgel LE, Crick FHC. Selfish DNA: the ultimate parasite. Nature. 1980;284 604-7.

3. Wicker T, Sabot F, Hua-Van A, Bennetzen IL, Capy P, Chalhoub B, et al. A unified classification system for eukaryotic transposable elements. Nat Rev Genet. 2007;8:973-82.

4. Lohe AR, Moriyama EN, Lidholm DA, Hartl DL. Horizontal transmission, vertical inactivation, and stochastic loss of mariner-like transposable elements. Mol Biol Evol. 1995;12:62-72.

5. Loreto ELS, Carareto CM a, Capy P. Revisiting horizontal transfer of transposable elements in Drosophila. Heredity (Edinb). 2008;100:545-54

6. Brennecke J, Aravin AA, Stark A, Dus M, Kellis M, Sachidanandam R, et al. Discrete small RNA-generating loci as master regulators of transposon activity in Drosophila. Cell. 2007;128:1089-103.

7. Aravin AA, Hannon GJ, Brennecke J. The piwi-piRNA pathway provides an adaptive defense in the transposon arms race. Science. 2007:318:761-4.

8. Maruyama K, Hartl DL. Evolution of the transposable element mariner in Drosophila species. Genet Soc Am. 1991;128:319-29.

9. Blumenstiel JP. Evolutionary dynamics of transposable elements in a small RNA world. Trends Genet Elsevier Ltd. 2011;27:23-31.

10. Groth SB, Blumenstiel JP. Horizontal transfer can drive a greater transposable element load in large populations. J Hered. 2017;108:36-44.

11. Gilbert C, Feschotte C. Horizontal acquisition of transposable elements and viral sequences: patterns and consequences. Curr Opin Genet Dev. 2018;49:15-24.

12. Daniels SB, Peterson KR, Strausbaugh LD, Kidwell MG, Chovnick A. Evidence for horizontal transmission of the $\mathrm{P}$ transposable element between Drosophila species. Genetics. 1990:124:339-55.

13. Hill T, Schlötterer C, Betancourt AJ. Hybrid dysgenesis in Drosophila simulans associated with a rapid invasion of the P-element. PLoS Genet. 2016;12

14. Kofler R, Hill T, Nolte V, Betancourt AJ, Schlötterer C. The recent invasion of natural Drosophila simulans populations by the P-element. Proc Natl Acad Sci U S A. 2015;112:6659-63.

15. Clark JB, Kidwell MG. A phylogenetic perspective on P transposable element evolution in Drosophila. Proc Nat Acad Sci USA. 1997;94:11428-33.

16. O'Brochta DA, Handler AM. Mobility of P elements in drosophilids and nondrosophilids. Proc Natl Acad Sci USA. 1988;85:6052-6.

17. Peccoud J, Loiseau V, Cordaux R, Gilbert C. Massive horizontal transfer of transposable elements in insects. Proc Natl Acad Sci. 2017;114:4721-6. 
18. Wallau GL, Vieira C, Loreto ÉLS. Genetic exchange in eukaryotes through horizontal transfer: connected by the mobilome. Mob DNA. 2018;9:1-16.

19. Dotto B, Carvalho E, Dezordi FZ, Pinto PM, de Campos TL, Rezende AM, et al. HTT-DB: new features and updates. Database. 2018;2018:1-12.

20. Schaack S, Gilbert C, Feschotte C. Promiscuous DNA: horizontal transfer of transposable elements and why it matters for eukaryotic evolution. Trends Ecol Evol. 2010;25:537-46.

21. Gilbert C, Schaack S, Pace JK II, Brindley PJ, Feschotte C. A role for hostparasite interactions in the horizontal transfer of DNA transposons across animal phyla. Nature. 2010;464:1347-50.

22. Capy P, Koga A, David JR, Hartl DL. Sequence analysis of active mariner elements in natural populations of Drosophila simulans. Genetics. 1992;130:499-506.

23. Hartl DL, Lozovskaya ER, Nurminsky DI, Lohe AR. What restricts the activity of mariner-like transposable elements? Trends Genet. 1997;13:197-201.

24. Kaplan N, Darden T, Langley CH. Evolution and extinction of transposable elements in Mendelian populations. Genetics. 1985;109:459-80.

25. Bartolomé C, Bello X, Maside X. Widespread evidence for horizontal transfer of transposable elements across Drosophila genomes. Genome Biol. 2009; 10:R22.

26. Hellen EHB, Brookfield JFY. The diversity of class II transposable elements in mammalian genomes has arisen from ancestral phylogenetic splits during ancient waves of proliferation through the genome. Mol Biol Evol. 2013;30:100-8.

27. Hellen EHB, Brookfield JFY. Transposable element invasions. Mob Genet Elements. 2013;3:e23920.

28. Petrov D a, Fiston-Lavier A-S, Lipatov M, Lenkov K, González J. Population genomics of transposable elements in Drosophila melanogaster. Mol Biol Evol. 2011;28:1633-44.

29. González J, Lenkov K, Lipatov M, Macpherson JM, Petrov D a. High rate of recent transposable element-induced adaptation in Drosophila melanogaster. PLoS Biol. 2008;6:e251.

30. Charlesworth B, Langley $\mathrm{CH}$. The population genetics of Drosophila transposable elements. Annu Rev Genet. 1989;23:251-87.

31. Sniegowski PD, Charlesworth B. Transposable element numbers in cosmopolitan inversions from a natural population of Drosophila melanogaster. Genetics. 1994;137:815-27.

32. Charlesworth B, Langley CH, Sniegowski PD. Transposable element distributions in Drosophila. Genetics. 1997;147:1993-5.

33. Clark AG, Eisen MB, Smith DR, Bergman CM, Oliver B, Markow T a, et al. Evolution of genes and genomes on the Drosophila phylogeny. Nature. 2007;450:203-18.

34. Sessegolo C, Burlet N, Haudry A, Biémont C, Vieira C, Tenaillon M, et al. Strong phylogenetic inertia on genome size and transposable element content among 26 species of flies. Biol Lett. 2016;12:521-4.

35. Dobzhansky TH, Hunter AS, Pavlovsky O, Spassky B, Wallace B. Genetics of natural populations. XXXI. Genetics of an isolated marginal population of Drosophila pseudoobscura. Genetics. 1963;48:91-103.

36. Dobzhansky T, Epling C. The suppression of crossing over in inversion heterozygotes of Drosophila pseudoobscura. Proc Natl Acad Sci USA. 1948; 34:137-41.

37. Patterson JT, Stone WS. Studies in the genetics of Drosophila. Austin: Univ Texas Publ; 1949. p. 7-17.

38. Palmieri N, Kosiol C, Schlötterer C. The life cycle of Drosophila orphan genes. elife. 2014;3:1-21.

39. Schaeffer SW, Anderson WW. Mechanisms of genetic exchange within the chromosomal inversions of Drosophila pseudoobscura. Genetics. 2005;171: 1729-39.

40. Dobzhansky T, Sturtevant AH. Inversions in chromosomes of Drosophila pseudoobscura. Genetics. 1937;23:28-64.

41. Dobzhansky T. Further data on the variation of the $Y$ chromosome in Drosophila pseudoobscura; 1937. p. 340-6.

42. Dobzhansky T. The Y chromosome of Drosophila Pseudoobscura. Genetics. 1935;20:366-76

43. Fuller ZL, Haynes GD, Richards S, Schaeffer SW. Genomics of Natural Populations: How Differentially Expressed Genes Shape the Evolution of Chromosomal Inversions in. Genetics. 2016;204:287-301.

44. Aquadro CF, Weaver AL, Schaeffer SW, Anderson WW. Molecular evolution of inversions in Drosophila pseudoobscura: the amylase gene region. Proc Natl Acad Sci U S A. 1991;88:305-9.

45. Noor M A F, Garfield D a, Schaeffer SW, Machado C a. Divergence between the Drosophila pseudoobscura and D. Persimilis genome sequences in relation to chromosomal inversions. Genetics 2007;177:1417-1428.
46. Machado C a, Haselkorn TS, Noor M a F. Evaluation of the genomic extent of effects of fixed inversion differences on intraspecific variation and interspecific gene flow in Drosophila pseudoobscura and Drosophila persimilis. Genetics. 2007;175:1289-306.

47. Crumpacker WBHDW, Ehrman L. Drosophila lowei, a new American member of the Obscura species group. Anim Entomol. 1969;62:388-93.

48. Dobzhansky TH. Drosophila miranda, a new species. Genetics. 1935;20:377-81.

49. Lachaise D, Silvain J-F. How two Afrotropical endemics made two cosmopolitan human commensals: the Drosophila melanogaster-D. simulans palaeogeographic riddle. Genetica. 2004;120:17-39.

50. Richards S, Liu Y, Bettencourt BR, Hradecky P, Letovsky S, Nielsen R, et al. Comparative genome sequencing of Drosophila pseudoobscura: chromosomal, gene, and cis-element evolution. Genome Res. 2005;15:1-18.

51. Kohany O, Gentles AJ, Hankus L, Jurka J. Annotation, submission and screening of repetitive elements in Repbase: RepbaseSubmitter and Censor. BMC Bioinformatics England. 2006;7:474.

52. Duff MO, Olson S, Wei X, Garrett SC, Osman A, Bolisetty M, et al. Genomewide identification of zero nucleotide recursive splicing in Drosophila. Nature. 2015;521:376-9. Nature Publishing Group, a division of Macmillan Publishers Limited. All Rights Reserved.

53. Ghildyal M, Zamore PD. Small silencing RNAs: an expanding universe. Nat Rev Genet. 2009;10:94-108.

54. Kelleher ES, Barbash D a. Analysis of piRNA-mediated silencing of active TEs in Drosophila melanogaster suggests limits on the evolution of host genome defense. Mol Biol Evol. 2013;30(8):1-14.

55. Malone CD, Brennecke J, Dus M, Stark A, Mccombie WR, Sachidanandam R, et al. Specialized piRNA pathways act in germline and somatic tissues of the Drosophila ovary. Cell. 2009;137:522-35.

56. Schneider TD, Stephens RM. Sequence logos: a new way to display consensus sequences. Nucleic Acids Res. 1990;18:6097-100.

57. Goubert C, Modolo L, Vieira C, Moro CV, Mavingui P, Boulesteix M. De novo assembly and annotation of the Asian tiger mosquito (Aedesalbopictus) repeatome with dnaPipeTE from raw genomic reads and comparative analysis with the yellow fever mosquito (Aedes aegypti). Genome Biol Evol. 2015;7:1192-205.

58. Tarailo-Graovac M, Chen N. Using RepeatMasker to identify repetitive elements in genomic sequences. Curr Protoc Bioinformatics. 2009;

59. Kofler R, Daniel G, Schlötterer C. PoPoolationTE2 : comparative population genomics of transposable elements using Pool-Seq. Mol Biol Evol. 2016:1-12.

60. Ellinghaus $D$, Kurtz S, Willhoeft U. LTRharvest, an efficient and flexible software for de novo detection of LTR retrotransposons. BMC Bioinformatics. 2008;9:18.

61. Ellison CE, Bachtrog D. Dosage compensation via transposable element mediated rewiring of a regulatory network. Science. 2013;342:846-50.

62. McGaugh SE, Heil CSS, Manzano-Winkler B, Loewe L, Goldstein S, Himmel $\mathrm{TL}$, et al. Recombination modulates how selection affects linked sites in Drosophila. PLoS Biol. 2012;10:1-17.

63. Gregory TR. Synergy between sequence and size in large-scale genomics. Nat Rev Genet. 2005;6:699-708.

64. Gregory TR, Johnston JS. Genome size diversity in the family Drosophilidae. Heredity (Edinb). 2008;101:228-38.

65. Bosco G, Campbell P, Leiva-Neto JT, Markow TA. Analysis of Drosophila species genome size and satellite DNA content reveals significant differences among strains as well as between species. Genetics. 2007;177:1277-90.

66. Francisco FO, Lemos B. How do Y-chromosomes modulate genome-wide epigenetic States : genome folding, chromatin sinks, and gene expression. J Genomics. 2014;2:94-103.

67. Burt A, Trivers R. Genes in. Conflict. 2006;

68. Zhou Q, Bachtrog D. Ancestral chromatin configuration constrains chromatin evolution on differentiating sex chromosomes in Drosophila. PLoS Genet. 2015;11:1-21.

69. Avila V, Marion de Procé S, Campos JL, Borthwick H, Charlesworth B, Betancourt AJ. Faster-X effects in two Drosophila lineages. Genome Biol Evol. 2014:6:2968-82.

70. Kofler R, Betancourt AJ, Schlötterer C. Sequencing of pooled DNA samples (Pool-Seq) uncovers complex dynamics of transposable element insertions in Drosophila melanogaster. PLoS Genet. 2012;8:1-16.

71. Bergman CM, Bensasson D. Recent LTR retrotransposon insertion contrasts with waves of non-LTR insertion since speciation in Drosophila melanogaster. Proc Natl Acad Sci U S A. 2007;104:11340-5.

72. Markow TA, O'Grady P. Drosophila: a guide to species identification. 2006. 
73. Yang Z. PAML 4: phylogenetic analysis by maximum likelihood. Mol Biol Evol. 2007;24:1586-91.

74. Wallau GL, Capy P, Loreto E, Le RA, Hua-Van A. VHICA, a new method to discriminate between vertical and horizontal transposon transfer: application to the mariner family with in Drosophila. Mol Biol Evol. 2016;33:1094-109.

75. Sturtevant a H, Dobzhansky T. Geographical distribution and cytology of "sex ratio" in Drosophila Pseudoobscura and related species. Genetics. 1936;21:473-90.

76. Tajima F. Statistical method for testing the neutral mutation hypothesis by DNA polymorphism. Genetics. 1989;123:585-95.

77. Hudson RR. Generating samples under a Wright-fisher neutral model of genetic variation. Bioinformatics. 2002;18:337-8.

78. Ernenwein L, Be A, Herniou EA, Cordaux R. Population genomics supports baculoviruses as vectors of horizontal transfer of insect transposons. Nat Commun. 2014;5:1-19.

79. Houck MA, Clark JB, Peterson KR, Kidwell MG. Possible horizontal transfer of Drosophila genes by the mite Protolaelaps regalis. Science. 1993;253:1125-9.

80. Bingham PM, Kidwell MG, Rubin GM. The molecular basis of P-M hybrid dysgenesis: the role of the P element, a P-strain-specific transposon family. Cell. 1982;29:995-1004

81. Kidwell JF, Kidwell MG, Sved JA. Hybrid dysgenesis in Drosophila melanogaster: a syndrome of aberrant traits including mutation, sterility, and male recombination. Genetics. 1977;86:813-33.

82. Smit AFA, Hubley R. RepeatModeler Open-1.0. 2008; Available from: www. repeatmasker.org

83. Price AL, Jones NC, P a P. De novo identification of repeat families in large genomes. Bioinformatics. 2005;21:351-8

84. Altschul SF, Gish W, Miller W, Myers EW, Lipman DJ. Basic local alignment search tool. J Mol Biol. 1990;215:403-10.

85. Kurtz $\mathrm{S}$. The Vmatch large scale sequence analysis software Features of Vmatch; 2010. p. 1-12.

86. Haas BJ, Papanicolaou A, Yassour M, Grabherr M, Blood PD, Bowden J, et al. De novo transcript sequence reconstruction from RNA-seq using the Trinity platform for reference generation and analysis. Nat Protoc. 2013;8:1494-512.

87. Katoh K, Misawa K, Kuma K, Miyata T. MAFFT: a novel method for rapid multiple sequence alignment based on fast Fourier transform. Nucleic Acids Res. 2002;30:3059-66.

88. Li H, Durbin R. Fast and accurate short read alignment with BurrowsWheeler transform. Bioinformatics. 2009;25:1754-60.

89. Quinlan AR, Hall IM. BEDTools: a flexible suite of utilities for comparing genomic features. Bioinformatics. 2010;26:841-2.

90. Kofler R, Daniel G, Schl C. PoPoolationTE2 : comparative population genomics of transposable elements using Pool-Seq; 2016. p. 1-7.

91. Leslie LK, Cohen JT, Newburger JW, Alexander ME, Wong JB, Sherwin ED, et al. Evolutionary flux of canonical microRNAs and mirtrons in Drosophila. Nat Genet. 2010;42:6-10.

92. Joshi N, Fass J. Sickle: A sliding window, adaptive, quality-based trimming tool for fastQ files, vol. 1; 2011. p. 33. Available from: https://github.com/ najoshi/sickle

93. Rahman R, Chirn G-W, Kanodia A, Sytnikova YA, Brembs B, Bergman CM, et al. Unique transposon landscapes are pervasive across Drosophila melanogaster genomes. Nucleic Acids Res. 2015;43:10655-72.

94. Brennecke J, Malone CD, Aravin AA, Sachidanandam R, Stark A, Hannon GJ. An epigenetic role for maternally inherited piRNAs in transposon silencing. Science. 2008;322:1387-92.

95. Obbard DJ, Gordon KHJ, Buck AH, Jiggins FM. The evolution of RNAi as a defence against viruses and transposable elements. Philos Trans R Soc Lond Ser B Biol Sci. 2009;364:99-115.

96. Levine MT, Malik HS. Learning to protect your genome on the fly. Cell. 2011; 147:1440-1. Elsevier Inc

97. DePristo MA, Banks E, Poplin R, Garimella KV, Maguire JR, Hartl C, et al. A framework for variation discovery and genotyping using next-generation DNA sequencing data. Nat Genet. 2011:43:491-8.

98. Guindon S, Dufayard J-F, Lefort V, Anisimova M, Hordijk W, Gascuel O. New algorithms and methods to estimate maximum-likelihood phylogenies: assessing the performance of PhyML 3.0. Syst Biol. 2010;59:307-21.

99. Kofler R, Orozco-terWengel P, de Maio N, Pandey RV, Nolte V, Futschik A, et al. Popoolation: a toolbox for population genetic analysis of next generation sequencing data from pooled individuals. PLoS One. 2011;6:e15925.

Ready to submit your research? Choose BMC and benefit from:

- fast, convenient online submission

- thorough peer review by experienced researchers in your field

- rapid publication on acceptance

- support for research data, including large and complex data types

- gold Open Access which fosters wider collaboration and increased citations

- maximum visibility for your research: over $100 \mathrm{M}$ website views per year

At BMC, research is always in progress.

Learn more biomedcentral.com/submissions 